\title{
Frequency of Ophthalmological Findings in Hospitalized COVID-19 Patients
}

\author{
Shayan Iqbal Khan ${ }^{1}$, FNU Versha ${ }^{2}$, Pooja Bai ${ }^{1}$, Parkash Bachani ${ }^{1}$, Muhammad Umair Nawaz ${ }^{3}$, Love
} Kumar $^{1}$, Sidra Naz ${ }^{4}$, Maha Jahangir ${ }^{5}$, Faizan Shaukat ${ }^{6}$

1. Internal Medicine, Liaquat University of Medical and Health Sciences, Jamshoro, PAK 2. Medicine and Surgery, Liaquat University of Medical and Health Sciences, Jamshoro, PAK 3. Internal Medicine, Jinnah Sindh Medical University, Karachi, PAK 4. Internal Medicine, University of Health Sciences (UHS), Lahore, PAK 5. Anesthesiology, Dow University of Health Sciences, Karachi, PAK 6. Internal Medicine, Dow University of Health Sciences, Karachi, PAK

Corresponding author: Shayan Iqbal Khan, shayan_7125@hotmail.com

\begin{abstract}
Introduction: The symptoms of coronavirus disease-19 (COVID-19) may range from mild to severe. Patients usually present with fever, cough, and other respiratory tract symptoms, but may also be asymptomatic. Some studies have also indicated the ocular involvement by the virus. This study aims to look deeply into all ophthalmic findings seen in COVID-19 patients and their clinical characteristics.
\end{abstract}

Methods: This longitudinal study was conducted in the COVID-19 unit of a tertiary care hospital, Pakistan. Data of patients hospitalized with COVID-19 infection between July 2020 and March 2021 were included in the study. Ophthalmological examination was done at the time of admission and was repeated every alternate day to look for any ophthalmological manifestation.

Results: Out of 441 ( $n=441), 61$ (13.8\%) participants had ophthalmological findings on examination. Patients with ophthalmological findings were significantly younger compared to patients without ophthalmological findings ( $42 \pm 6$ years vs. $44 \pm 7$; p-value, 0.03 ). C-reactive protein (CRP) was also significantly higher in patients with ophthalmological findings (122.2 \pm 16.2 vs. $112.8 \pm 19.8$; p-value, 0.005$)$. The most common ophthalmological finding was conjunctival irritation (50.8\%), followed by diplopia (27.8\%) and cotton wool spots (27.8\%).

Conclusion: Ophthalmological findings are prevalent in patients with COVID-19. In this study, patients with higher CRP levels were associated with ophthalmological findings. It is important to conduct ophthalmological examinations in patients with COVID-19, as they may give a clue about other complications associated with COVID-19.

Review began 05/01/2021 Review ended 05/09/2021 Published 05/10/2021

\section{() Copyright 2021}

Khan et al. This is an open access article distributed under the terms of the Creative Commons Attribution License CC-BY 4.0., which permits unrestricted use, distribution, and reproduction in any medium, provided the original author and source are credited.
Categories: Internal Medicine, Ophthalmology, Infectious Disease

Keywords: covid-19, ophthalmological manifestation, corona virus disease, pakistan, clinical characteristics

\section{Introduction}

The coronavirus disease-19 (COVID-19) was declared a public health emergency by the World Health Organization (WHO) in early 2020, after the outbreak of the severe acute respiratory syndrome coronavirus 2 (SARS-CoV-2) in China [1]. The virus spread quickly to involve other parts of the world, leading to a pandemic, which not only affected the global economy but also led to almost 2.9 million deaths worldwide in its first year $[1,2]$.

The symptoms of COVID-19 may range from mild to severe. Patients usually present with fever, cough, shortness of breath, dyspnea, and other respiratory tract symptoms, but may also be asymptomatic. In the more severe cases, patients may develop severe respiratory disease and pneumonia, which may require intensive care assistance and usually have poor recovery rates [3]. This variation in presentation is poorly understood but the individual host response to the virus may have a role in determining the severity of the disease [4].

Apart from the respiratory tract, COVID-19 is also seen to affect other organ systems resulting in gastrointestinal, cardiovascular, neurological, hematological, and other symptoms. Some studies have also indicated the ocular involvement by the virus [5]. The COVID-19 can affect both the ocular surfaces and the deeper neural tissue. Studies have shown the presence of SARS-CoV-2 in the conjunctival swabs [6]. The usual ocular surface symptoms are that of conjunctival irritation like itching, chemosis, tearing, and foreign body sensation. The presence of these symptoms is seen to correlate with disease severity [6-8]. The retinal involvement with abnormal findings on dilated eye examination has also been reported in COVID-19 patients. Usual abnormalities seen on the exam include ischemia with cotton wool spots and pallor, increased tortuosity, and dilation of vessels as well as the presence of flame-shaped hemorrhages $[9,10]$. This ocular involvement raises concerns regarding disease transmission between physicians and patients 


\section{Cureus}

during ophthalmic consultation and also highlights the need to consider long-term detrimental effects on the vision, attributed to the retinal changes, seen in these patients. Therefore, this study aims to look deeply into all ophthalmic findings seen in COVID-19 patients and their implication on general health and disease transmission.

\section{Materials And Methods}

This longitudinal study was conducted in the COVID-19 unit of a tertiary care hospital, Pakistan. Data of patients hospitalized with COVID-19 infection between July 2020 and March 2021 were included in the study. All subjects gave their informed consent for inclusion before they participated in the study. Ethical board approval was taken before the start of the study. Patients were treated as per local guideline for the treatment of COVID-19 [11]. Patients with diabetes and hypertension were excluded from the study to avoid including pre-existing ophthalmological manifestation.

Ophthalmological examination, including fundoscopy and tonometry, was done at the time of admission and was repeated every alternate day to look for any ophthalmological manifestation till patient discharge or their death. Patients' age, gender, lab reports, need for ventilation and ophthalmological findings were recorded in a self-structured questionnaire.

The collected data were analyzed using Statistical Package for Social Sciences ${ }^{\circledR}$ software version 23.0 (SPSS; IBM Corp., Armonk, NY, USA). Mean and standard deviation (SD) were calculated for numerical data. Frequency and percentage were calculated for categorical data. Frequencies were compared using Chisquare. Independent $\mathrm{t}$-test and chi-square were used as appropriate. A p-value less than 0.05 meant that there is a difference between the two groups and the null hypothesis is void.

\section{Results}

A total number of patients included in the study were $441(n=441) .61$ participants had ophthalmological findings on examination. Patients with ophthalmological findings were significantly younger compared to patients without ophthalmological findings (42 \pm 6 years vs. $44 \pm 7$; p-value, 0.03 ). C-reactive protein (CRP) was also significantly higher in patients with ophthalmological findings (122.2 \pm 16.2 vs. $112.8 \pm 19.8$; pvalue, 0.005) (Table 1).

\begin{tabular}{|c|c|c|c|}
\hline Characteristics (Mean + SD) & Patients with ophthalmological findings $(n=61)$ & Patients without ophthalmological findings $(n=380)$ & P-value \\
\hline CRP (mg/L) & $122.2 \pm 16.2$ & $112.8 \pm 19.8$ & $0.005^{*}$ \\
\hline Oxygen saturation (\%) & $85.2 \pm 4.2$ & $86.1 \pm 4.9$ & $0.17^{\star \star}$ \\
\hline Need for ventilation & $11(18.0 \%)$ & $61(16.0 \%)$ & $0.15^{\star \star}$ \\
\hline Death & $5(8.1 \%)$ & $24(6.3 \%)$ & $0.07^{\star \star}$ \\
\hline
\end{tabular}

TABLE 1: Clinical and laboratory characteristics in patients with and without ophthalmologic findings

BPM: Breath per minute; CRP: C-reactive protein; LDH: Lactate dehydrogenase; SOC: Standard of care. SD: Standard deviation

*: significant, **: not significant

The most common ophthalmological finding was conjunctival irritation (50.8\%), followed by diplopia (27.8\%) and cotton wool spots (27.8\%) (Table 2). 


\section{Cureus}

\begin{tabular}{|l|l|}
\hline Ophthalmological findings $(\mathbf{n}=\mathbf{6 1})$ & Number of patients (\%) \\
\hline Conjunctival irritation & $31(50.8 \%)$ \\
Diplopia & $17(27.8 \%)$ \\
Cotton wool spots & $17(27.8 \%)$ \\
Increased intraocular pressure & $15(24.5 \%)$ \\
Micro-haemorrhages & $12(19.6 \%)$ \\
Ophthalmoparesis & $11(18.0 \%)$ \\
Papilledema & $06(9.83 \%)$ \\
Infectious keratitis & $04(6.5 \%)$ \\
Subconjunctival haemorrhage & $02(3.2 \%)$ \\
\hline
\end{tabular}

TABLE 2: Ophthalmological manifestations in COVID-19 patients

\section{Discussion}

In our study, participants bearing ophthalmological findings were significantly younger than those who did not bear any. Patients with ophthalmological manifestations showed elevated levels of CRP with a higher respiratory rate. Among the ophthalmological findings, conjunctival irritation was the most common, followed by diplopia and cotton wool spots.

A study conducted by Wu et al. showed that patients with ocular signs and symptoms showed higher levels of CRP with symptoms of conjunctivitis, which were also observed in our study [6]. Many studies have reported that inflammatory markers, such as CRP, play a major role in triggering inflammation [12]. In patients with COVID-19, studies have shown that the majority of them report high levels of inflammatory markers [13,14]. CRP starts elevating within 46 hours of the onset of inflammation and has a half-life of 4-7 hours [15]. This is why it can be used to track active inflammation, such as conjunctivitis, in our case. Moreover, CRP status is also directly linked with the width of lung lesions and the severity of clinical symptoms of the COVID-19 patients [16].

Humans are infected by seven types of coronaviruses (CoVs), namely human coronavirus 229E (HCoV-229E), human coronavirus NL63 (HCoV-NL63), human coronavirus OC43 (HCoV-OC43), human coronavirus HKU1 (HCoV-HKU1), middle east respiratory syndrome coronavirus (MERS-CoV), severe acute respiratory syndrome coronavirus (SARS-CoV), and severe acute respiratory syndrome coronavirus- 2 (SARS-CoV-2) [17]. Most of them are known to cause respiratory infections, but they are also reported to affect the gastrointestinal system and ocular tissues [18,19]. Out of these, NL63 and SARS-CoV-2 are believed to affect the ocular system. Between 2000 and 2003, reports of bronchiolitis and conjunctivitis have been noted due to NL63 [20]. Additionally, in 2004, SARS-CoV-RNA was found in tear specimens of three patients, pointing towards the presence of virus in tears [18]. Theories were proposed stating the possibility of the conjunctiva being the exposed site of the attack by the pathogenic droplets; route of infection from the airway tract through the nasolacrimal duct, or hematogenous infection of the lacrimal gland, were put forward [18]. Analyses based on genome and structure show similarity in the receptor binding sites between SARS-CoV and SARS-CoV-2, with alike pathology and epidemiology $[21,22]$. Therefore, a similar effect on ocular tissues is expected. However, ophthalmic manifestations have been reported by very few people $[6,8]$.To the best of our knowledge, this is the first study from this region that have studied ophthalmological manifestation in COVID-19 patients. Since the study was conducted in one institute, the sample size was might not have been diverse and care should be taking inferring the result to a greater population. Another limitation might be that this study only included hospitalized patients and does not include mild and moderate cases of COVD-19.

The above-stated literature and the findings of our study suggest that patients with ocular manifestations should be dealt with precautions, and screening tests should be done under the supervision of doctors to avoid further complications. Moreover, more clinical studies involving a larger sample size should be concluded to further evaluate and explore ophthalmic presentation in COVID-19 patients.

\section{Conclusions}

Ophthalmological findings are prevalent in patients with COVID-19. In this study, patients with ophthalmological findings are associated with higher CRP. It is important to conduct ophthalmological examinations in patients with COVID-19, as they may give a clue or help prevent other complications 
associated with COVID-19. This study also suggests that long-term follow-up of patients with COVID-19 should also include an ophthalmological examination.

\section{Additional Information \\ Disclosures}

Human subjects: Consent was obtained or waived by all participants in this study. Liaquat University of Medical and Health Sciences issued approval LUMHS/2020/COVID/ERC-11. Animal subjects: All authors have confirmed that this study did not involve animal subjects or tissue. Conflicts of interest: In compliance with the ICMJE uniform disclosure form, all authors declare the following: Payment/services info: All authors have declared that no financial support was received from any organization for the submitted work. Financial relationships: All authors have declared that they have no financial relationships at present or within the previous three years with any organizations that might have an interest in the submitted work. Other relationships: All authors have declared that there are no other relationships or activities that could appear to have influenced the submitted work.

\section{References}

1. Jee Y: WHO International Health Regulations Emergency Committee for the COVID-19 outbreak. Epidemiol Health. 2020, 42:e2020013. 10.4178/epih.e2020013

2. Chakraborty C, Sharma AR, Sharma G, Bhattacharya M, Lee SS: SARS-CoV-2 causing pneumonia-associated respiratory disorder (COVID-19): diagnostic and proposed therapeutic options. Eur Rev Med Pharmacol Sci. 2020, 24:4016-2. 10.26355/eurrev_202004_20871

3. Grasselli G, Pesenti A, Cecconi M: Critical care utilization for the COVID-19 outbreak in Lombardy, Italy: early experience and forecast during an emergency response. JAMA. 2020, 323:1545-6. 10.1001/jama.2020.4031

4. Mehta P, McAuley DF, Brown M, Sanchez E, Tattersall RS, Manson JJ: COVID-19: consider cytokine storm syndromes and immunosuppression. Lancet. 2020, 395:1033-4. 10.1016/S0140-6736(20)30628-0

5. Lai CC, Ko WC, Lee PI, Jean SS, Hsueh PR: Extra-respiratory manifestations of COVID-19. Int J Antimicrob Agents. 2020, 56:106024. 10.1016/j.ijantimicag.2020.106024

6. Wu P, Duan F, Luo C, Liu Q, Qu X, Liang L, Wu K: Characteristics of ocular findings of patients with Coronavirus disease 2019 (COVID-19) in Hubei Province, China. JAMA Ophthalmol. 2020, 138:575-8. 10.1001/jamaophthalmol.2020.1291

7. Zhou Y, Duan C, Zeng Y, et al.: Ocular findings and proportion with conjunctival SARS-COV-2 in COVID-19 patients. Ophthalmology. 2020, 127:982-3. 10.1016/j.ophtha.2020.04.028

8. Xia J, Tong J, Liu M, Shen Y, Guo D: Evaluation of coronavirus in tears and conjunctival secretions of patients with SARS-CoV-2 infection. J Med Virol. 2020, 92:589-94. 10.1002/jmv.25725

9. Pereira LA, Soares LCM, Nascimento PA, et al.: Retinal findings in hospitalised patients with severe COVID19. Br J Ophthalmol. 2020, 2020:317576. 10.1136/bjophthalmol-2020-317576

10. Invernizzi A, Torre A, Parrulli S, et al.: Retinal findings in patients with COVID-19: Results from the SERPICO-19 study. EClinicalMedicine. 2020, 27:100550. 10.1016/j.eclinm.2020.100550

11. Clinical Management Guidelines for COVID-19 Infections . (2020). Accessed: April 26, 2021: https://storage.covid.gov.pk/new_guidelines/11December2020_20201211_Clinical_Management_Guidelines_for_COVID19_infec....

12. Jeandrot A, Richard JL, Combescure C, et al.: Serum procalcitonin and C-reactive protein concentrations to distinguish mildly infected from non-infected diabetic foot ulcers: a pilot study. Diabetologia. 2008, 51:34752. 10.1007/s00125-007-0840-8

13. Tan C, Huang Y, Shi F, et al.: C-reactive protein correlates with computed tomographic findings and predicts severe COVID-19 early. J Med Virol. 2020, 92:856-62. 10.1002/jmv.25871

14. Li R, Tian J, Yang F, et al.: Clinical characteristics of 225 patients with COVID-19 in a tertiary hospital near Wuhan, China. J Clin Virol. 2020, 127:104363. 10.1016/j.jcv.2020.104363

15. Gabay C, Kushner I: Acute-phase proteins and other systemic responses to inflammation . N Engl J Med. 1999, 340:448-54. 10.1056/NEJM199902113400607

16. Wang L: C-reactive protein levels in the early stage of COVID-19 . Med Mal Infect. 2020, 50:332-4. 10.1016/j.medmal.2020.03.007

17. Corman VM, Muth D, Niemeyer D, Drosten C: Hosts and sources of endemic human coronaviruses . Adv Virus Res. 2018, 100:163-88. 10.1016/bs.aivir.2018.01.001

18. Loon SC, Teoh SC, Oon LL, Se-Thoe SY, Ling AE, Leo YS, Leong HN: The severe acute respiratory syndrome coronavirus in tears. Br J Ophthalmol. 2004, 88:861-3. 10.1136/bjo.2003.035931

19. Yeo C, Kaushal S, Yeo D: Enteric involvement of coronaviruses: is faecal-oral transmission of SARS-CoV-2 possible?. Lancet Gastroenterol Hepatol. 2020, 5:335-7. 10.1016/S2468-1253(20)30048-0

20. Vabret A, Mourez T, Dina J, et al.: Human coronavirus NL63, France. Emerg Infect Dis. 2005, 11:1225-9. 10.3201/eid1108.050110

21. Seah I, Agrawal R: Can the coronavirus disease 2019 (COVID-19) affect the eyes? A review of coronaviruses and ocular implications in humans and animals. Ocul Immunol Inflamm. 2020, 28:391-5. 10.1080/09273948.2020.1738501

22. Wan Y, Shang J, Graham R, Baric RS, Li F: Receptor recognition by the novel coronavirus from Wuhan: an analysis based on decade-long structural studies of SARS coronavirus. J Virol. 2020, 94:00127-20. 10.1128/JVI.00127-20 